\title{
BOUNDS FOR HOLOMORPHIC VECTOR FIELDS
}

E. R. FERNHOLZ ${ }^{1}$

1. Introduction. In this paper we find bounds for holomorphic vector fields on an analytic space. The bounds are given with respect to the seminorms defined on the holomorphic functions by compact subsets. Thus global holomorphic vector fields are continuous as operators on the Frechet space of global holomorphic functions.

The bounds we find are designed to prove that certain subdomains of analytic spaces are holomorphically convex. We give an example of this in $\S 3$. The bounds can be used to prove essentially the following statement: if a holomorphically convex analytic space has enough global vector fields, then domains of holomorphy in it are also holomorphically convex.

We define an analytic space to be a connected ringed space $\left(X, \mathcal{O}_{X}\right)$ such that each $x \in X$ has a neighborhood $U$ with $\left(U, \theta_{X} \mid U\right)$ isomorphic (as a ringed space) to $\left(V, \Theta_{V}\right)$ where $V$ is a closed analytic subvariety of $C^{n}$, for some $n$ depending on $x$, and $\mathcal{\theta}_{V}=\mathcal{\theta}_{C^{n}} / \mathscr{g} \mid V$ where $g$ is the sheaf of ideals of $V$. By this definition, all analytic spaces are reduced. We shall not assume that the analytic spaces we deal with are irreducible, of pure dimension, or that they have finite maximum dimension.

We shall abbreviate $\left(X, \mathcal{O}_{X}\right)$ by $X$ if no confusion is possible. $S(X)$ will denote the set of singular points of $X . H(X)=H^{0}\left(X, \mathcal{O}_{X}\right)$ is the algebra of global holomorphic functions on $X . \theta_{X, x}$ is the stalk of $\theta_{X}$ at $x \in X$.

2. Bounds for holomorphic vector fields. First we shall define vector fields on an analytic space $X$ (after Rossi [2]).

Definition 2.1. Let $X$ be an analytic space and $x \in X$. A tangent vector to $X$ at $x$ is a mapping $t: \Theta_{X, x} \rightarrow C$ such that for $a, b \in C, f, g \in \mathcal{\Theta}_{X, x}$ :

(i) $t(a f+b g)=a t(f)+b t(g)$,

(ii) $t(f g)=f(x) t(g)+g(x) t(f)$.

The tangent space to $X$ at $x$, denoted by $T_{X, x}$, is the set of all tangent vectors to $X$ at $x$. It is a vector space.

Definition 2.2. Let $X$ be an anlytic space. A holomorphic vector field on $X$ is a function $t: x \mapsto T_{X, x}$ defined for all $x \in X$ such that $f \in H(X)$ implies that the function $g$ defined by $g(x)=t(x) f_{x}$ is also

Received by the editors September 12, 1968 and, in revised form, December 18, 1968.

1 This work supported in part by the National Science Foundation. 
holomorphic (where $f_{x}$ is the germ of $f$ at $x$ ). Let $\Im_{X}$ represent the sheaf of germs of holomorphic vector fields on $X$.

After this we shall write $t f$ for the function defined by $t(x) f_{x}$. Note that, on manifolds, Definition 2.2 is equivalent to the standard definition of vector field.

The following two propositions are due to Rossi [2].

Proposition 2.3. Let $X$ be an analytic space and $\mathfrak{J}_{X}$ the sheaf of germs of holomorphic vector fields on $X$. Then $\mathfrak{J}_{X}$ is a coherent analytic sheaf.

Proposition 2.4. Let $D$ be a domain of holomorphy in $\mathrm{C}^{n}$ and $V a$ closed subvariety of $D$. If $t$ is a holomorphic vector field on $V$, then there exists a holomorphic vector field $\tilde{t}$ on $D$ such that for any $\tilde{f} \in H(D)$, $f=\tilde{f} \mid V$, we have

$$
(\tilde{t} \tilde{f}) \mid V=t f .
$$

$\tilde{t}$ will be called an extension of $t$ to $D$.

We shall be dealing with iterates of vector fields. Notice that the above definition of extension is compatible with iteration in the following sense: If $t_{1}, t_{2}$ are vector fields on $V$ and $\tilde{t}_{1}, \tilde{t}_{2}$ are their extensions to $D$ ( $V$ and $D$ as in Proposition 2.4, and all vector fields assumed to be holomorphic) then

$$
\left(\tilde{t}_{1} \tilde{t}_{2} \tilde{f}\right) \mid V=t_{1}\left(\left(\tilde{t}_{2} \tilde{f}\right) \mid V\right)=t_{1} t_{2}(\tilde{f} \mid V)=t_{1} t_{2} f
$$

for all $\tilde{f} \in H(D), f=\tilde{f} \mid V$.

The vector fields on $D$ are generated by the partial derivatives $\partial / \partial z_{i}, i=1, \cdots, n$. A general vector field on $D$ has the form

$$
\sum_{i=1}^{n} a_{i} \frac{\partial}{\partial z_{i}}, \quad a_{i} \in H(D), \quad i=1, \cdots, n .
$$

We shall need the following proposition, proved in Gunning and Rossi [1, p. 157].

Proposition 2.5. Let $U$ be a neighborhood of the origin in $\mathbf{C}^{n}$, and $V$ a subvariety of $U$. There are polydiscs centered at the origin (in suitable coordinates), $\Delta \subset \Delta^{\prime} \subset U$, and a number $m>0$ such that every function $f$, holomorphic on $V \cap \bar{\Delta}^{\prime}$, has an extension $\tilde{f}$ to $\Delta$ such that

$$
\|\tilde{f}\|_{\Delta} \leqq m\|f\|_{\bar{\Delta}^{\prime} \cap v} .
$$

We are now prepared to prove the existence of bounds for holomorphic vector fields. For simplicity, first we shall prove a proposition for a single vector field and then consider the case of iterated vector fields. 
Proposition 2.6. Let $X$ be an analytic space and $t$ a holomorphic vector field on $X$. Let $K$ be a compact subset of $X$. Then there exist a compact set $K^{\prime} \subset X$ and a constant $c$ such that for all $f \in H(X)$

$$
\|t f\|_{K} \leqq c\|f\|_{K^{\prime}}
$$

Proof. For each $x \in K$ find a neighborhood $U_{x}^{\prime}$ of $x$ which is biholomorphic to a subvariety $V_{x}$ of $\boldsymbol{C}^{n_{x}}$. Such neighborhoods exist for an appropriate integer $n_{x}$ since $X$ is an analytic space. Let $\phi_{x}: U_{x}^{\prime} \rightarrow V_{x}$ be the biholomorphic mapping with $\phi_{x}(x)=0 \in C^{n_{x}}$. For each $V_{x}$ use Proposition 2.5 to find polydiscs $\Delta_{x} \subset \Delta_{x}^{\prime}$ in $C^{n_{x}}$ such that, for any $g \in H\left(V_{x}\right)$, there is an extension $\tilde{g}$ of $g$ to $\Delta_{x}^{\prime}$ and a constant $m_{x}$ such that

$$
\|\tilde{g}\|_{\Delta_{x}} \leqq m_{x}\|g\|{ }_{\bar{\Delta}_{x} \cap V_{x}}^{\prime} .
$$

The sets $U_{x}=\phi_{x}^{-1}\left(\Delta_{x} \cap V_{x}\right)$ form an open cover for $K$, so we can extract a finite subcover $\left\{U_{x_{i}}\right\}$. To simplify notation, we shall now use the subscript $i$ to replace $x_{i}$, so the cover is now $\left\{U_{i}\right\}$. The corresponding sets $K_{i}=\phi_{i}^{-1}\left(\bar{\Delta}_{i}^{\prime} \cap V_{i}\right)$ are compact, so $K^{\prime}=\bigcup K_{i}$ is also compact.

If $g$ is any function holomorphic on $U_{i}^{\prime}$, then $g_{i}=g \circ \phi_{i}{ }^{-1}$ is holomorphic on $V_{i}$. For any holomorphic vector field $s$ on $U_{i}^{\prime}$ we can define a vector field $s_{i}$ on $V_{i}$ by $s_{i} g_{i}=(s g) \circ \phi_{i}{ }^{-1}$ for all $g \in H\left(U_{i}^{\prime}\right)$.

For $\epsilon>0, z_{0} \in C^{n}$, let $\Delta\left(z_{0}, \epsilon\right)=\left\{z \in C^{n}:\left|z_{j}-z_{0_{j}}\right|<\epsilon, j=1, \cdots, n\right\}$. Then we can choose $\epsilon>0$ sufficiently small that for any $x \in K$, $\Delta\left(\phi_{i}(x), \epsilon\right) \subset \Delta_{i}$ for some $i$.

Now let $f \in H(X)$, so $f$ is holomorphic on each $U_{i}^{\prime}$. On each $V_{i}$ define $f_{i}$ as above and let $\tilde{f}_{i}$ be the extension to $\Delta_{i}^{\prime}$ satisfying $\left(^{*}\right)$. Let $t_{i}$ be the vector field on $V_{i}$ defined (as above) by $t$ on $U_{i}^{\prime} \subset X$. Let $\tilde{t}_{i}$ be an extension of $t_{i}$ to $\Delta_{i}^{\prime}$. Using the Cauchy integral formula we see that for $x \in K$ there is an $i$ such that

$$
\left|\tilde{t}_{i} \tilde{f}_{i}\left(\phi_{i}(x)\right)\right| \leqq\left(c_{i} / \epsilon\right)\left\|\tilde{f}_{i}\right\|_{\Delta\left(\phi_{i}(x), \epsilon\right)}
$$

where

$$
\begin{aligned}
\tilde{t}_{i} & =\sum_{j=1}^{n_{i}} \gamma_{j} \frac{\partial}{\partial z_{j}}, \quad \gamma_{j} \in H\left(\Delta_{i}^{\prime}\right), \\
c_{i} & =n_{i} \max _{j}\left\|\gamma_{j}\right\|_{\Delta\left(\phi_{i}(x), \epsilon\right) .}
\end{aligned}
$$

Now we have $\left\|\tilde{f}_{i}\right\|_{\Delta\left(\phi_{i}(x), \epsilon\right)} \leqq\left\|\tilde{f}_{i}\right\|_{\Delta_{i} \leqq m_{i}}\left\|f_{i}\right\|_{\Delta_{i}^{\prime} \cap V_{i}} \leqq m_{i}\|f\|_{K^{\prime}}$, and since $\tilde{t}_{i} \tilde{f}_{i} \mid V_{i}=t_{i} f_{i}$, we have $\left|t_{i} f_{i}\left(\phi_{i}(x)\right)\right| \leqq\left(c_{i} m_{i} / \epsilon\right)\|f\|_{K^{\prime}}$. Since $t_{i} f_{i}\left(\phi_{i}(x)\right)$ $=t f(x),|t f(x)| \leqq\left(c_{i} m_{i} / \epsilon\right)\|f\|_{K^{\prime}}$. Now if $c=\max _{i} c_{i} m_{i} / \epsilon$, then $|t f(x)|$ $\leqq c\|f\|_{K^{\prime}}$ for all $x \in K$ or $\|t f\|_{K} \leqq c\|f\|_{K^{\prime}}$. 
Now we shall extend this proposition to include iterated vector fields.

Proposition 2.7. Let $X$ be an analytic space and $K \subset X$ a compact subset. Let $t_{1}, \cdots, t_{r}$ be holomorphic vector fields on $X$ and $t^{k}$ any $k$-fold iterate of the $t_{i}$. Then there exist a compact set $K^{\prime} \subset X$ and constants $c, m$ such that for all $f \in H(X)$ and for all integers $k>0$

$$
\left\|t^{k} f\right\|_{K} \leqq c^{k} m(k+1) !\|f\|_{K^{\prime}}
$$

Proof. Choose $U_{j}^{\prime}, U_{j}, v_{j}, \Delta_{j}, \Delta_{j}^{\prime}, C^{n_{i}}, \phi_{j}, f_{j}, \tilde{f}_{j}, K^{\prime}, \epsilon$ exactly as in the proof of Proposition 2.6. Let $t_{i j}$ be the vector field on $V_{j}$ defined by $t_{i}$ on $U_{j}^{\prime}$. Let $\tilde{t}_{i j}$ be an extension of $t_{i j}$ to $C^{n_{i}}$. Let $N=\max _{j} n_{j}$ (where $V_{j} \subset C^{n_{j}}$ ). For convenience, we can assume all the $n_{j}=N$, embedding $\boldsymbol{C}^{\boldsymbol{n}_{j}}$ in $\boldsymbol{C}^{N}$ if necessary. Then we can write

$$
\tilde{t}_{i j}=\sum_{p=1}^{N} \alpha_{i j p} \frac{\partial}{\partial z_{p}}, \quad \alpha_{i j p} \in H\left(C^{N}\right),
$$

where the $z_{p}, p=1, \cdots, N$, are the coordinates in $C^{N}$. We shall abbreviate this by writing

$$
\tilde{t}_{i j}=\sum_{p=1}^{N} \alpha \frac{\partial}{\partial z} .
$$

Since we shall eventually take a maximum of all the $\alpha_{i j p}$ this will not matter. Let $t_{j}^{k}$ be the representative of $t^{k}$ in $V_{j}$ and $\tilde{t}_{j}^{k}$ the extension of $t_{j}^{k}$ to $C^{N}$. Then

$$
\begin{aligned}
\tilde{t}_{j}^{k} \tilde{f}_{j}= & \sum_{p_{1}, \cdots, p_{k}=1}^{N} \alpha^{k} \frac{\partial^{k} \tilde{f}_{j}}{\partial z^{k}}+\sum_{p_{1}, \cdots, p_{k}=1}^{N} k \alpha^{k-1} \frac{\partial \alpha}{\partial z} \frac{\partial^{k-1} \tilde{f}_{j}}{\partial z^{k-1}}+\cdots \\
& +\sum_{p_{1}, \cdots, p_{k}=1}^{N}\left[\alpha\left(\frac{\partial \alpha}{\partial z}\right)^{k-1}+\cdots+\alpha^{k-1} \frac{\partial^{k-1} \alpha}{\partial z^{k-1}}\right] \frac{\partial \tilde{f}_{j}}{\partial z} .
\end{aligned}
$$

For any $x \in K$ there is a $j$ such that $\Delta\left(\phi_{j}(x), \epsilon\right) \subset \Delta_{j}$ and by the Cauchy integral formula

$$
\begin{aligned}
\left|\tilde{t}_{j}^{k} \tilde{f}_{j}\left(\phi_{j}(x)\right)\right| \leqq N^{k}|\alpha|^{k} \frac{k !}{\epsilon^{k}}\left\|\tilde{f}_{j}\right\|_{\Delta\left(\phi_{j}(x), \epsilon\right)} & +N^{k} k|\alpha|^{k-1} \frac{|\alpha|}{\epsilon} \frac{(k-1) !}{\epsilon^{k-1}}\left\|\tilde{f}_{j}\right\|_{\Delta\left(\phi_{j}(x), \epsilon\right)}+\cdots \\
& +N^{k}\left(|\alpha|^{k} \frac{k !}{\epsilon^{k-1}}\right) \frac{1}{\epsilon}\left\|\tilde{f}_{j}\right\|_{\Delta\left(\phi_{j}(x), \epsilon\right)}
\end{aligned}
$$


where

$$
|\alpha|=\sup _{i j p}\left\{\left|\alpha_{i j p}(z)\right|: z \in \Delta_{j}, i=1, \cdots, k ; p=1, \cdots, N\right\} .
$$

Then

$$
\begin{aligned}
\left|t^{k} f(x)\right| & \leqq N^{k}|\alpha|^{k} \frac{(k+1) !}{\epsilon^{k}}\left\|\tilde{f}_{j}\right\|_{\Delta\left(\phi_{j}(x), \epsilon\right)} \\
& \leqq N^{k}|\alpha|^{k} \frac{(k+1) !}{\epsilon^{k}} m_{j}\|f\|_{K^{\prime}} .
\end{aligned}
$$

Let $c=N|\alpha| / \epsilon, m=\max _{j} m_{j}$, then

$$
\left\|t^{k} f\right\|_{K} \leqq c^{k} m(k+1) !\|f\|_{K^{\prime}} .
$$

3. Domains of holomorphy. In this section we apply Proposition 2.7 to prove certain facts about domains of holomorphy on analytic spaces. First we shall recall some definitions.

Definition 3.1. Let $X$ be an analytic space and $K$ a compact subset of $X$. The holomorphic hull of $K, \hat{K}$, is defined by $\hat{K}=\{z \in X$ : $\left.|f(z)| \leqq\|f\|_{K}, f \in H(X)\right\} . X$ is holomorphically convex if for any compact subset $K \subset X, \hat{K}$ is also compact.

Definition 3.2. Let $X$ be an analytic space and $D$ a connected open subset. $D$ is a domain of holomorphy if for any $x \in \operatorname{bd} D$ there is a function $f \in H(D)$ such that $f$ cannot be continued analytically over $x$. This means that for any neighborhood $U$ of $x, f \mid U \cap D$ cannot be extended to $U$.

Proposition 3.3. Let $X$ be an analytic space such that $H^{0}\left(X, \Im_{X}\right)$ generates the stalks of $J_{X}$ at all regular points of $X$. Let $D$ be a domain of holomorphy in $X$. For $K \subset D, K$ compact, let $\hat{K}=\left\{z \in D:|f(z)| \leqq\|f\|_{K}\right.$, $f \in H(D)\}$. Then bd $\hat{K} \cap$ bd $D \subset S(X)$.

(The boundaries are as subsets of $\mathrm{X}$.)

Proof. Let $N$ be an open neighborhood of $S(X)$. Then $X-N$ is nonsingular. Suppose there is a point $x \in \operatorname{bd} D \cap b d \hat{K} \cap(X-N)$. Assume that $x$ lies in a component of $X-N$ of dimension $n(X-N$ may have components of different dimensions). Let $U$ be a neighborhood of $x$ such that $U \cap S(X)=\varnothing$ and such that there are local coordinates in $U, z_{1}, \cdots, z_{n}$, with associated partial derivatives

$$
\frac{\partial}{\partial z_{i}}=\sum_{r=1}^{n} \beta_{i r} t_{r}
$$

where $t_{r} \in H^{0}\left(X, \Im_{X}\right), \beta_{i r} \in H^{0}\left(U, \theta_{X}\right) ; i, r=1, \cdots, n$. Assume that the $\beta_{\text {ir }}$ are bounded on $U$, making $U$ smaller if necessary. 
As in Proposition 2.7 we can find a neighborhood $U^{\prime}$ of $x$, relatively compact in $U$, and constants $c_{1}, m_{1}$ such that

$$
\left\|t^{p} \beta\right\|_{U^{\prime}} \leqq \stackrel{p}{c_{1} m_{1}(p+1) !\|\beta\|_{U}}
$$

where $\beta$ is any of the $\beta_{i r}$ and $t^{p}$ is any $p$-fold iterate of the $t_{r}$.

Now let $f \in H(D)$ be a function which cannot be continued over $x$. For any $y \in U^{\prime} \cap \hat{K}$ we can express a $k$ th order partial derivative (with indices omitted) of $f$ near $y$ as follows:

$$
\begin{aligned}
\frac{\partial^{k} f}{\partial z^{k}}=\sum_{r_{1}, \cdots, r_{k}=1}^{n} \beta^{k}\left(t^{k} f\right) & +\sum_{r_{1}, \cdots, r_{k=1}}^{n} k \beta^{k-1}(t \beta)\left(t^{k-1} f\right)+\cdots \\
& +\sum_{r_{1}, \cdots, r_{k}=1}^{n}\left[\beta(t \beta)^{k-1}+\cdots+\beta^{k-1}\left(t^{k-1} \beta\right)\right](t f) .
\end{aligned}
$$

From this we obtain

$$
\begin{aligned}
\left|\frac{\partial^{k} f(y)}{\partial z^{k}}\right| \leqq n^{k}|\beta|^{k}\left|t^{k} f(y)\right|+n^{k} k|\beta|^{k-1}|t \beta|\left|t^{k-1} f(y)\right|+\cdots \\
+n^{k}\left[|\beta||t \beta|^{k-1}+\cdots+|\beta|^{k-1}\left|t^{k-1} \beta\right|\right]|t f(y)|
\end{aligned}
$$

where $\left|t^{p} \beta\right|=\left\|t^{p} \beta\right\|_{U^{\prime}}, p=0, \cdots, k$.

By Proposition 2.7 we can find constants $c_{2}, m_{2}$ and a compact set $K^{\prime} \subset D$ such that

$$
\left|t^{p} f(y)\right| \leqq\left\|t^{p} f\right\|_{\hat{K}}=\left\|t^{p} f\right\|_{K} \leqq \stackrel{p}{c_{2} m_{2}}(p+1) !\|f\|_{K^{\prime}}
$$

for $t^{p}$ any $p$-fold iterate of the $t_{r}$. If we let $m=\max \left(m_{1}, m_{2}\right)$ and $c=\max \left(c_{1}, c_{2}\right)$ we obtain

$$
\left|\frac{\partial^{k} f(y)}{\partial z^{k}}\right| \leqq 2 n^{k} c^{k} m^{k}|\beta|^{k}(k+2) !\|f\|_{K^{\prime}}
$$

Thus the Taylor series for $f$ converges in a neighborhood (of fixed radius) of $y$. Since $y$ can be chosen arbitrarily close to $x, f$ is holomorphic at $x$ and we have a contradiction. Therefore there is no $x \in \operatorname{bd} D \cap \operatorname{bd} \hat{K} \cap(X-N)$. Since $N$ was arbitrary, bd $D \cap \operatorname{bd} \hat{K} \subset S(X)$.

Proposition 3.4. Let $X, D$, and $K$ be as in Proposition 3.3. Assume either that $X$ is holomorphically convex or that $D$ is relatively compact. Suppose bd $D \cap S(X)=\varnothing$. Then $D$ is holomorphically convex.

Proof. Let $\hat{K}^{x}=\left\{z \in X:|f(x)| \leqq\|f\|_{k}, f \in H(X)\right\}$. If $X$ is holomorphically convex, $\hat{K}^{\boldsymbol{x}}$ is compact; if not then $\bar{D}$ is compact by hypothesis. Let $W=\hat{K}^{x}$ or $\bar{D}$, whichever is compact. $\hat{K} \subset W$, so it 
suffices to show that $\hat{K}$ is closed in $X . \hat{K}$ is closed in $D$ and since bd $\hat{K} \cap \operatorname{bd} D \subset \operatorname{bd} D \cap S(X)=\varnothing$, we have that $\hat{K}$ is closed in $X$.

Corollary 3.5. A domain of holomorphy $D$ in a Stein space $X$ such that bd $D \cap S(X)=\varnothing$ is holomorphically convex (and thus a Stein space itself).

Corollary 3.6. A domain of holomorphy in a Stein manifold is holomorphically convex (and thus a Stein manifold itself).

Corollary 3.7. A domain of holomorphy in $P^{n}$, complex projective $n$-space, is holomorphically convex.

Proof of Corollaries 3.5, 3.6, and 3.7. In each case $H^{0}\left(X, \jmath_{X}\right)$ generates the stalks of $J_{X}$ and all the spaces are holomorphically convex, so Proposition 3.4 applies.

REMARK. The hypothesis that bd $D \cap S(X)=\varnothing$ is necessary. For bd $D \cap S(X) \neq \varnothing$, Rossi has an example of a domain of holomorphy in a Stein space which is not holomorphically convex (see [3]). We do not know what happens in the case of a holomorphically convex manifold which does not have enough global vector fields to satisfy the hypotheses of Proposition 3.3.

\section{BIBLIOGRAPHY}

1. R. Gunning and H. Rossi, Analytic functions of several complex variables, Prentice-Hall, Englewood Cliffs, N. J., 1965.

2. H. Rossi, Vector fields on analytic spaces, Ann. of Math. (2) 78 (1963), 455-467.

3. - Picard variety of an isolated singular point, mimeographed notes, Brandeis University, Waltham, Mass., 1967.

UNIVERSITY OF WASHINGTON 